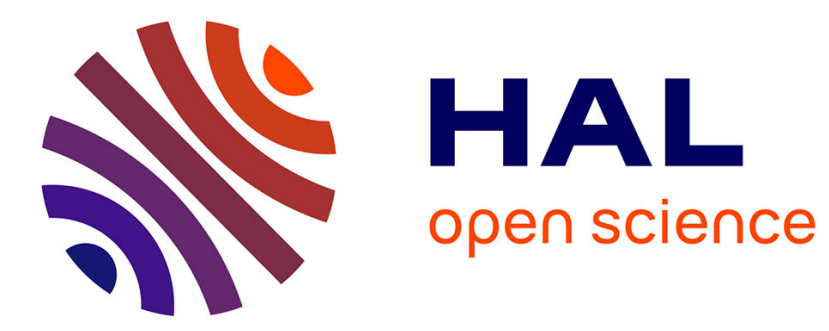

\title{
Prescribed-time predictor control of LTI systems with distributed input delay
}

Salim Zekraoui, Nicolás Espitia, Wilfrid Perruquetti

\section{To cite this version:}

Salim Zekraoui, Nicolás Espitia, Wilfrid Perruquetti. Prescribed-time predictor control of LTI systems with distributed input delay. 60th IEEE Conference on Decision and Control, Dec 2021, Austin (virtual), United States. hal-03514452

\section{HAL Id: hal-03514452 \\ https://hal.archives-ouvertes.fr/hal-03514452}

Submitted on 6 Jan 2022

HAL is a multi-disciplinary open access archive for the deposit and dissemination of scientific research documents, whether they are published or not. The documents may come from teaching and research institutions in France or abroad, or from public or private research centers.
L'archive ouverte pluridisciplinaire HAL, est destinée au dépôt et à la diffusion de documents scientifiques de niveau recherche, publiés ou non, émanant des établissements d'enseignement et de recherche français ou étrangers, des laboratoires publics ou privés. 


\title{
Prescribed-time predictor control of LTI systems with distributed input delay
}

\author{
Salim Zekraoui
}

Nicolás Espitia

Wilfrid Perruquetti

\begin{abstract}
This paper deals with prescribed-time stabilization of controllable linear systems with distributed input delay. We model the input delay as a transport PDE and reformulate the original problem as a cascade PDE-ODE system while accounting for the infinite dimensionality of the actuator. We build on reduction-based and backstepping-forwarding transformations to convert the system into a target system having the prescribed-time stability property. Then, we prove the bounded invertibility of the transformations and hence we show that the prescribed-time stability property is preserved into the original problem. To better illustrate the ideas of this approach, we focus first on the scalar case. Then, we give a sketch of the main lines for the general case. To this end, we choose the ODE dynamics of the target system to be a Linear Time-Varying system so that we can rely on recent developments which include a polynomial-based Vandermonde matrix and the generalized Laguerre polynomials that allow a compact formulation for the stability analysis. A simulation example is presented to illustrate the obtained results.
\end{abstract}

\section{INTRODUCTION}

During the last decade, several research contributions have been reported on non-asymptotic concepts such as finite-time, fixed-time, and prescribed-time stabilization and estimation for linear and nonlinear ordinary differential equations (ODEs) (see e.g. [15], [4], [26], [22], [27], [17], [19], to mention a few).

Finite-time convergence refers to a terminal time which depends on the system's initial conditions, whereas the terminal time for fixed-time convergence is independent on the system's initial conditions insofar as the terminal time is lower bounded by a bounded function of the initial conditions. More recently, the prescribed-time convergence concept has arisen to deal with a more demanding type of convergence, which allows the terminal time to be prescribed independently of initial conditions and parameters [27], [17], [19], [29], [16], [31]. This type of convergence has emerged for tactical and strategic missile guidance problems but many numerous other applications (e.g. rendezvous, spacecraft docking, trajectory tracking for nonholonomic mobile robots, finite-time deployment and formation control for multi-agent systems, weather forecasting) require the transient process must occur within a given time.

For partial differential equations (PDEs), these nonasymptotic concepts have become an attractive research area since PDEs describe many complex systems (thermal and

\footnotetext{
*This work has been partially supported by ANR Project Finite4SoS (ANR 15-CE23-0007). S. Zekraoui, N. Espitia and W. Perruquetti are with CRIStAL UMR 9189 CNRS - Centre de Recherche en Informatique Signal et Automatique de Lille - CNRS, Centrale Lille, Univ. Lille, F-59000 Lille, France. Corresponding author: salim.zekraouidcentralelille.fr
}

fluid dynamics, chemical reactions, batteries, etc). For instance, finite-time stabilization has been studied in, e.g., [7], [9]. For linear parabolic PDEs, null controllability/finite-time stabilization [8] and prescribed-time stabilization [14], [28] have been achieved by using the backstepping approach with time-varying kernels.

Time-delay systems are ubiquitous in engineering, where delays can appear e.g., in the inputs (point-wise or distributed) [23], [32], or in the outputs and network graph communication topologies as in [30]. For linear systems with input delays, exponential stabilization is performed based on predictor feedback. In [21], [20], under an ODEPDE cascade setting, the classical predictor is related to the backstepping approach. The backstepping PDE framework for time-delay systems has been extended to deal with delay-adaptive control, delay compensation and estimation problems, nonlinear systems with input delay, time-varying delays, and distributed input delays [6], [5], [2]. Nevertheless, results for finite-, fixed- and prescribed-time concepts for time-delay systems remain sparse. Some pioneering contributions on finite/fixed-time stability of time-delay systems are [11], [18], [25]; more recent results are [10], [33], [24] and [12], [13], where the latter deals with prescribed-time predictor control for LTI systems with input delay. Although, to best of our knowledge, prescribed-time stabilization for LTI systems with distributed input delay has not been studied yet in the literature.

In this paper, we combine the ideas of [34], [17], and [13] to handle the problem of estimation of LTI systems in the presence of distributed input delay. We model the input delay as a transport PDE and reformulate the original problem as a cascade PDE-ODE system while accounting for the infinite dimensionality of the actuator. We build on the reductionbased and backstepping-forwarding transformations to transform the system into a target system having the prescribedtime stability property. We relate back such a property through a suitable study of the bounded invertibility of the aforementioned transformations. The resulting predictorlike feedback is made up of time-varying gains. To better illustrate the ideas of this approach, we focus first on the scalar case. Then, we give a sketch of the main lines for the general case.

This paper is organized as follows. In Section II, we introduce the LTI system with distributed input delay. In Section III, we use a PDE-ODE setting and a suitable transformations to come up with a prescribed-time predictor controller. We focus on the scalar case to better communicate the main ideas of our approach. Then, an overview of the 
generalization to a LTI systems is provided in Section IV. In Section $\mathrm{V}$ we consider a numerical example to illustrate the results. Finally, conclusions and perspectives are given in Section VI.

Notations: We denote by $\mathbb{R}_{+}$the set of nonnegative real numbers. For non zero integers $m$ and $n$, let $J_{n}=$ $\left(\left(0_{(n-1) \times 1}, I_{n-1}\right)^{\top}, 0_{n \times 1}\right)^{\top}$ (Jordan matrix) and $\mathcal{L}_{n}(p)=$ $\left(0_{(n) \times(n-1)}, p\right)^{\top}$, where $0_{m \times n}$ is the $(m, n)$-zero matrix which is the matrix with all entries equal to zero, $I_{m}$ be the identity matrix of dimension $m$, and $p \in \mathbb{R}^{n} . L_{m}^{(\alpha)}(\cdot)$ denotes the generalized Laguerre polynomials and $\bar{\sigma}_{n}(\cdot)$ denotes the elementary symmetric polynomials. We denote by $L^{2}\left((0, h), \mathbb{R}^{n}\right)$ the set of all functions $f:[0, h] \rightarrow \mathbb{R}^{n}$ such that $\int_{0}^{h} f(x)^{2} d x<\infty$, and for simplicity, we will use the notation $L^{2}$ instead of $L^{2}\left((0, h), \mathbb{R}^{n}\right)$.

\section{Problem Statement}

We consider the following controllable linear system with distributed input delay as stated in [34]:

$$
\dot{X}(t)=A X(t)+\int_{0}^{h} B(h-\sigma) u(t-\sigma) d \sigma,
$$

where $X(t) \in \mathbb{R}^{n}$ is the system state, $u(\theta) \in \mathbb{R}$ for $\theta \in[t-h, t]$ is the actuator state, $u(t) \in \mathbb{R}$ is the control input, $h>0$ is known constant delay, and $A, B$ are the system matrix and input vector of appropriate dimensions, respectively. The input vector $B(\cdot)$ is a continuous realvalued vector function defined on $[0, h]$.

The objective of this paper is to design a predictor-like feedback achieving "uniform fixed-time stability in a prescribed time" (in short UPrTS) in the same spirit as in [17], but whose specific notion is adapted to the current problem (which is infinite dimensional) as we will see in Section III-A.2 and Section IV-A.1. In order to better communicate the key ideas in our approach, we are going to deal first with the analysis and design for a simple linear scalar equation with distributed input delay.

\section{PRESCRIBED TIME PREDICTOR CONTROL: A PDE-ODE SETTING AND REDUCTION-BASED AND BACKSTEPPING-FORWARDING TRANSFORMATIONS}

We reformulate the system (1) into a cascade PDEODE setting (i.e. cascade linear hyperbolic PDE with an LTI system) introduced in [3], [34], and that employed a Backstepping-forwarding transformation, and a reductionbased change of variable. As in [12], the main idea of our approach is to transform the original system into a target system that is UPrTS (in an appropriate sense) and that we choose to satisfy the property of convergence in a prescribed time $T+h+t_{0}$. Here, $T$ is fixed a priori, $h$ is the known input delay and for simplicity of notations, we take the initialization time $t_{0}=0$.

As previously mentioned, in an attempt to better illustrate the key ideas of our method, we first deal with a scalar linear system with distributed input delay. The generalization to the $n$-dimensional case (i.e. LTI systems of the form (1)) follows the same strategy like the one we used in the scalar case (which represent the heart of this paper contribution) as well as some suitable changes of variables and some transformations in the framework of linear timevarying systems. Due to space limitation, only an outline of the extension is given, in a very informative way, in Section IV.

\section{A. Scalar case}

Let us consider the following scalar control system:

$$
\dot{X}(t)=a X(t)+b \int_{0}^{h} u(t-\sigma) d \sigma .
$$

which is a particular case of (1) in the problem statement. Following [34], the system (2) can be rewritten as PDE-ODE system:

$$
\begin{aligned}
\dot{X}(t) & =a X(t)+b \int_{0}^{h} \omega(t, \sigma) d \sigma \\
\omega_{t}(t, x) & =\omega_{x}(t, x) \\
\omega(t, h) & =u(t),
\end{aligned}
$$

$t \geq 0, \sigma$ and $x$ belong to $[0, h] . \omega(t, \cdot)$ is the transport PDE state whose solution is given by $\omega(t, x)=u(t+x-h)=$ $u(t-\sigma)$, where $\sigma=h-x$. We aim at stabilizing (3)-(5) (in turn (2)) in a prescribed time $T+h$.

1) Reduction-based and backstepping-forwarding transformation: We consider the following reduction-based change of variables:

$$
Z(t)=X(t)+\int_{0}^{h} q(\sigma) \omega(t, \sigma) d \sigma .
$$

where $q(\cdot)$ and its domain are yet to be characterized while meeting the property $q(0)=0$. In addition, consider the following backstepping-forwarding transformation:

$$
\zeta(t, x)=\omega(t, x)-\gamma(t, x) Z(t),
$$

where the function $\gamma$ is time-varying. The inverse transformation is given as follows:

$$
\omega(t, x)=\zeta(t, x)+\gamma(t, x) Z(t) .
$$

Under (6) and (7), we want to transform (3) into the following target system:

$$
\begin{aligned}
\dot{Z}(t) & =-c(t) Z(t), \\
\zeta_{t}(t, x) & =\zeta_{x}(t, x), \\
\zeta(t, h) & =0,
\end{aligned}
$$

where $\zeta:[0, \infty) \times[0, h] \rightarrow \mathbb{R}$ is the transport PDE state and $c$ is given by the following blow-up function :

$$
c(t)=\frac{c_{0}^{2} T^{2}}{(T-t)^{2}}, \quad c(0)=c_{0}^{2} .
$$

Following the standard approach to find the kernel equations, we can prove that the time-varying function $\gamma$ and the function $q$ satisfy the following PDE system:

$$
\begin{gathered}
q^{\prime}(\sigma)+a q(\sigma)=b \\
\gamma_{x}(t, x)-\gamma_{t}(t, x)=(a+\gamma(t, h) q(h)) \gamma(t, x),
\end{gathered}
$$


where $q$ and $\gamma$ are defined on the domains, respectively $\mathcal{T}_{q}$ : $\{\sigma: 0 \leq \sigma \leq h\}$ and $\mathcal{T}_{\gamma}:\{(t, x): 0 \leq x \leq h, \quad 0 \leq t<$ $T+x-h\}$.

Proposition 1: The system (13)-(14) has well-posed $\mathcal{C}^{\infty}$ solutions on $\mathcal{T}_{q}$ and $\mathcal{T}_{\gamma}$, given by

$$
\begin{gathered}
q(\sigma)=\frac{b}{a}\left(1-e^{-a \sigma}\right), \\
\gamma(t, x)=-\frac{a(a+c(t-h+x))}{b\left(1-e^{-a h}\right)} e^{c_{0} T(\sqrt{c(t)}-\sqrt{c(t+x-h)})},
\end{gathered}
$$

where $c$ is defined by (12).

Proof: From (13), since $q(0)=0$, we find $q(\sigma)=$ $\left.\frac{b}{a}\left(1-e^{-a(\sigma)}\right)\right)$. Concerning (14), we know, thanks to the chosen $Z$-dynamics of target system (9), that

$$
\begin{aligned}
\dot{Z}(t) & =-c(t) Z(t)=\dot{X}(t)+\int_{0}^{h} q(\sigma) \omega_{t}(t, \sigma) d \sigma \\
& =a X(t)+a \int_{0}^{h} q(\sigma) \omega(t, \sigma) d \sigma+q(h) \gamma(t, h) Z(t) \\
& =a Z(t)+q(h) \gamma(t, h) Z(t)
\end{aligned}
$$

then, we get

$$
-c(t)=a+q(h) \gamma(t, h)
$$

Using (18) and the following change of variables in (14)

$$
\gamma(t, x)=e^{\int_{0}^{t} c(s) d s} \Gamma(t, x),
$$

we get $\Gamma_{x}(t, x)-\Gamma_{t}(t, x)=0$, whose solution is obtained by the method of characteristics as follows:

$\Gamma(t, x)=\Gamma(t-h+x, h)=e^{-\int_{0}^{t+x-h} c(s) d s} \gamma(t-h+x, h)$, then,

$$
\begin{aligned}
\gamma(t, x) & =\gamma(t-h+x, h) e^{-\int_{t}^{t+x-h} c(s) d s} \\
& =\gamma(t-h+x, h) e^{c_{0} T(\sqrt{c(t)}-\sqrt{c(t+x-h)})} .
\end{aligned}
$$

Now, let us calculate $\gamma(t-h+x, h)$. From (18) we get

$$
\gamma(t, h)=\frac{-a-c(t)}{q(h)}=\frac{-a-c(t)}{\frac{b}{a}\left(1-e^{-a h}\right)},
$$

which finally yields

$$
\gamma(t, x)=\frac{-(a+c(t+x-h))}{\frac{b}{a}\left(1-e^{-a h}\right)} e^{c_{0} T(\sqrt{c(t)}-\sqrt{c(t+x-h)}) .}
$$

Prescribed-time predictor control: From (7) and (6), at $x=h$, and using (15), (16) the boundary control is then,

$$
u(t)=\frac{-(a+c(t))}{\frac{b}{a}\left(1-e^{-a h}\right)}\left(X(t)+\frac{b}{a} \int_{0}^{h}\left(1-e^{-a \sigma}\right) \omega(t, \sigma) d \sigma\right),
$$

2) Stability analysis: We first study the stability of the target system and then we establish the bounded invertibility of the transformations by a suitable norm equivalence.

Lemma 1: Let $c$ be given by (12) with $c_{0}, T>0$ fixed. Let $h>0$ be a known delay. Then, the $Z$-dynamics of target system (9) satisfies, for any $Z_{0} \in \mathbb{R}$ and for all $t \in[0, T)$,

$$
|Z(t)|^{2} \leq \eta_{z} e^{-2 c_{0} T \sqrt{c(t)}}\left|Z_{0}\right|^{2} \cdot{ }^{1}
$$

where $\eta_{z}=e^{2 T c_{0}^{2}}$. In particular, $|Z(t)|^{2} \rightarrow 0$, as $t \rightarrow T$ and $|Z(t)| \equiv 0$, for $t \geq T$.

Moreover, the transport PDE $\zeta$ of target system (9) is fixedtime stable and for any $\zeta(0, x) \in L^{2}((0, h), \mathbb{R})$, it holds that $\|\zeta(t, \cdot)\| \equiv 0$ for all $t \geq h$.

Proposition 2: For the transformations (6) and (7), the following estimates hold:

$$
\begin{aligned}
\|\omega(t, \cdot)\|_{L^{2}}^{2} \leq & 2\|\zeta(t, \cdot)\|_{L^{2}}^{2}+2\|\gamma(t, \cdot)\|_{L^{2}}^{2}|Z(t)|^{2} \\
|X(t)|^{2} \leq & \left(2+4\|\gamma(t, .)\|_{L^{2}}^{2}\|q\|_{L^{2}}^{2}\right)|Z(t)|^{2} \\
& +4\|q\|_{L^{2}}^{2}\|\zeta(t, \cdot)\|_{L^{2}}^{2} .
\end{aligned}
$$

Proof: On one hand, from (7), we have

$$
|\omega(t, x)| \leq|\zeta(t, x)|+|\gamma(t, x)||Z(t)|,
$$

then $|\omega(t, x)|^{2} \leq 2|\zeta(t, x)|^{2}+2|\gamma(t, x)|^{2}|Z(t)|^{2}$,

from which (24) is obtained. On the other hand, by the Cauchy-Schwarz inequality, we get

$$
\begin{aligned}
|X(t)| & \leq|Z(t)|+\int_{0}^{h}|q(\sigma)||\omega(t, \sigma)| d \sigma, \\
& \leq|Z(t)|+\|q\|_{L^{2}}\|\omega(t, .)\|_{L^{2}} .
\end{aligned}
$$

Using the Young's inequality, we obtain

$$
|X(t)|^{2} \leq 2|Z(t)|^{2}+2\|q\|_{L^{2}}^{2}\|\omega(t, .)\|_{L^{2}}^{2},
$$

which combined with (24) leads to (25).

Lemma 2: Let $\gamma$ be given by (16). Then, the following holds true:

$$
\lim _{t \rightarrow T+h}\|\gamma(t, \cdot)\|_{L^{2}}^{2} e^{-2 c_{0} T \sqrt{c(t)}}=0 .
$$

Proof: Let $I(t)=\|\gamma(t, \cdot)\|_{L^{2}}^{2} e^{-2 c_{0} T \sqrt{c(t)}}$. Using (16), we obtain

$$
\begin{aligned}
& I(t)=\int_{0}^{h} e^{-2 c_{0} T \sqrt{c(t)}}|\gamma(t, x)|^{2} d x \\
& =\int_{0}^{h} e^{-2 c_{0} T \sqrt{c(t)}} \frac{a^{2}(a+c(t+x-h))^{2}}{b^{2}\left(1-e^{-a h}\right)^{2}} e^{2 c_{0} T(\sqrt{c(t)}-\sqrt{c(t-h+x)})} d x \\
& \leq 2 \delta \int_{0}^{h}\left(a^{2}+c(t+x-h)^{2}\right) e^{-2 c_{0} T \sqrt{c(t-h+x)}} d x \\
& \leq F_{1}(t-h)+F_{2}(t-h),
\end{aligned}
$$

where $\delta=\frac{a^{2}}{b^{2}\left(1-e^{-a h}\right)^{2}}$, and

$$
\begin{aligned}
& F_{1}(t-h)=2 \delta a^{2} \int_{0}^{h} e^{-2 c_{0} T \sqrt{c(t-h+x)}} d x \\
& F_{2}(t-h)=2 \delta \int_{0}^{h} c(t+x-h)^{2} e^{-2 c_{0} T \sqrt{c(t-h+x)}} d x .
\end{aligned}
$$

${ }^{1} e^{-2 c_{0} T \sqrt{c(t)}}$ is a monotonically decreasing smooth "bump-like" function having the property $e^{-2 c_{0} T \sqrt{c(t)}} \equiv 0, \forall t \geq T$ (see e.g. [28]). 
It can be shown that $F_{1}(t-h)$ and $F_{2}(t-h)$ goes to zero in time $T+h$. Then, $I(t) \rightarrow 0$ as $t \rightarrow T+h$.

Proposition 3: Let $q$ be given by (15). Then, the following holds true:

$$
\left|Z_{0}\right|^{2} \leq 2\left(1+\|q\|_{L^{2}}^{2}\right)\left(\left|X_{0}\right|^{2}+\|\omega(0, .)\|_{L^{2}}^{2}\right) .
$$

Proof: By replacing $t=0$ in (6) and by the CauchySchwarz inequality, we get

$$
\begin{aligned}
\left|Z_{0}\right| & \leq\left|X_{0}\right|+\int_{0}^{h} \mid q(\sigma)\|\omega(0, \sigma)\| d \sigma \\
& \leq\left|X_{0}\right|+\|q\|_{L^{2}}\|\omega(0, .)\|_{L^{2}}
\end{aligned}
$$

by the Young's inequality, we obtain

$$
\left|Z_{0}\right|^{2} \leq 2\left|X_{0}\right|^{2}+2\|q\|_{L^{2}}^{2}\|\omega(0, .)\|_{L^{2}}^{2}
$$

from which (30) is deduced.

Theorem 1: Let $c$ be given by (12). Let $h>0, c_{0}>0$, $T>0$ fixed. Let $\gamma$ be given by (16). Then, the solution of the closed-loop system (3) with prescribed-time predictor control (22) is UPrTS in the following sense: for any $X_{0} \in \mathbb{R}$ and $\omega(0, \cdot) \in L^{2}((0, h), \mathbb{R})$, the quantity $|X(t)|^{2}+\|\omega(t, \cdot)\|_{L^{2}}^{2}$ remains bounded for $t \in[0, \max \{T, h\}]$, and for all $t \in$ $[\max \{T, h\}, T+h)$, the following estimate holds:

$$
\begin{aligned}
|X(t)|^{2}+\|\omega(t, \cdot)\|_{L^{2}}^{2} \leq \eta_{z} M e^{-2 c_{0} T \sqrt{c(t)}}\|\gamma(t, \cdot)\|_{L^{2}}^{2} \\
\times\left(\left|X_{0}\right|^{2}+\|\omega(0, \cdot)\|_{L^{2}}^{2}\right)
\end{aligned}
$$

with $M=4\left(1+2\|q\|_{L^{2}}^{2}\right)\left(1+\|q\|_{L^{2}}^{2}\right)$. In particular,

$$
|X(t)|^{2}+\|\omega(t, \cdot)\|_{L^{2}}^{2} \rightarrow 0, \quad \text { as } \quad t \rightarrow T+h
$$

Moreover, $|u(t)| \rightarrow 0$ as $t \rightarrow T$.

Proof: By Proposition 2 we have for all $t \in[0, T+h)$

$$
|X(t)|^{2}+\|\omega(t, \cdot)\|_{L^{2}}^{2} \leq M_{1}\|\zeta(t, \cdot)\|_{L^{2}}^{2}+M_{2}(t)|Z(t)|^{2},
$$

with $M_{1}=2+4\|q\|_{L^{2}}^{2}$ and $M_{2}(t)=2+M_{1}\|\gamma(t, \cdot)\|_{L^{2}}^{2}$. Assume $T \geq h$. By the fact that $\|\zeta(t, \cdot)\|_{L^{2}} \rightarrow 0$ as $t \rightarrow h$, then it holds for $t \in[h, T+h)$,

$$
\begin{aligned}
|X(t)|^{2}+\|\omega(t, \cdot)\|_{L^{2}}^{2} & \leq M_{2}(t)|Z(t)|^{2} \\
& =2|Z(t)|^{2}+M_{1}\|\gamma(t, \cdot)\|_{L^{2}}^{2}|Z(t)|^{2},
\end{aligned}
$$

and by Lemma 1 , we have $|Z(t)| \equiv 0$ for $t \geq T$, then it holds for $t \in[T, T+h)$,

$$
\begin{aligned}
|X(t)|^{2}+\|\omega(t, \cdot)\|_{L^{2}}^{2} & \leq M_{1}\|\gamma(t, \cdot)\|_{L^{2}}^{2}|Z(t)|^{2} \\
& \leq\left|Z_{0}\right|^{2} \eta_{z} M_{1} e^{-2 c_{0} T \sqrt{c(t)}}\|\gamma(t, \cdot)\|_{L^{2}}^{2}
\end{aligned}
$$

Notice that $\|\gamma(t, \cdot)\|_{L^{2}}^{2}|Z(t)|^{2}$ does not vanish when $t \rightarrow$ $T$, even though $Z(t)$ vanishes. This is because, the rate of the growth-in-time of $\gamma(t, \cdot)$ is the same the rate of the decreasing-time of $Z$, on the interval $[0, T]$.

On the other hand if $T \leq h$, we use the fact that $|Z(t)| \equiv 0$ for $t \geq T$, then it holds for $t \in[T, T+h)$,

$$
|X(t)|^{2}+\|\omega(t, \cdot)\|_{L^{2}}^{2} \leq M_{1}\left(\|\zeta(t, \cdot)\|_{L^{2}}^{2}+\|\gamma(t, \cdot)\|_{L^{2}}^{2}|Z(t)|^{2}\right),
$$

Using the fact that $\|\zeta(t, \cdot)\|_{L^{2}} \rightarrow 0$ as $t \rightarrow h$, the inequality (35) holds for $t \in[h, T+h)$. Using Proposition 3, we obtain

$$
\begin{array}{r}
|X(t)|^{2}+\|\omega(t, \cdot)\|_{L^{2}}^{2} \leq \eta_{z} M e^{-2 c_{0} T \sqrt{c(t)}}\|\gamma(t, \cdot)\|_{L^{2}}^{2} \\
\times\left(\left|X_{0}\right|^{2}+\|\omega(0, .)\|_{L^{2}}^{2}\right),
\end{array}
$$

where $M=M_{1}\left(2+2\|q\|_{L^{2}}^{2}\right)$. We finally obtain by Lemma 2 , that $|X(t)|^{2}+\|\omega(t, \cdot)\|_{L^{2}}^{2} \rightarrow 0$, as $t \rightarrow T+h$. It remains to show that $|u(t)| \rightarrow 0$. Indeed, from the transformation (7) it holds

$$
|u(t)|=|\gamma(t, h)||Z(t)| \leq \frac{a|a+c(t)|}{b\left|1-e^{-a}\right|} \sqrt{\eta_{z}}\left|Z_{0}\right| e^{-c_{0} T \sqrt{c(t)}},
$$

from which we can conclude that $|u(t)| \rightarrow 0$ as $t \rightarrow T$.

\section{EXTENSION TO LTI SYSTEMS WITH SINGLE DISTRIBUTED INPUT DELAY}

In this section, we present a sketch of the results regarding the general case for the design of the predictor-feedback prescribe time stabilization of (1). Let us consider the cascade PDE-ODE formulation of (1), i.e.

$$
\begin{aligned}
\dot{X}(t) & =A X(t)+\int_{0}^{h} B(\sigma) \omega(t, \sigma) d \sigma, \\
\omega_{t}(t, x) & =\omega_{x}(t, x), \\
\omega(t, h) & =u(t) .
\end{aligned}
$$

Let $\bar{B}_{h}=\int_{0}^{h} e^{-A(h-y)} B(y) d y$. We assume that the pair $\left(A, \bar{B}_{h}\right)$ is controllable:

Assumption 1: The controllability matrix $\mathbf{C}_{h}=$ $\left[\bar{B}_{h}, A \bar{B}_{h}, \ldots, A^{n-1} \bar{B}_{h}\right]$ is of full rank $n$.

1) Reduction-based and backstepping-forwarding transformation: We consider the following reduction-based change of variables inspired from [34]:

$$
Z(t)=P\left(X(t)+\int_{0}^{h} \int_{0}^{\sigma} e^{-A(\sigma-y)} B(y) d y \omega(t, \sigma) d \sigma\right)
$$

where $P=\left[g^{\top},(g A)^{\top}, \ldots,\left(g A^{n-1}\right)^{\top}\right]^{\top}$, and $g$ the $n$-th row of the matrix $\mathbf{C}_{h}^{-1}$ (existence of the inverse comes from Assumption 1).

In addition, consider the following backstepping-forwarding transformation

$$
\zeta(t, x)=\omega(t, x)-\gamma^{\top}(t, x) Z(t),
$$

where the function $\gamma$ is time-varying. such that $\gamma^{\top}(t, x) Z(t)=\sum_{i=1}^{n} \gamma_{i}(t, x) Z_{i}(t)$.

Then, the system (37) is mapped into the following target system:

$$
\begin{aligned}
\dot{Z}(t) & =C(t) Z(t), \\
\zeta_{t}(t, x) & =\zeta_{x}(t, x), \\
\zeta(t, h) & =0,
\end{aligned}
$$

where $C(t)$ is a companion canonical matrix, i.e. $C(t)=$ $J_{n}+\mathcal{L}_{n}(-p(t))$, where $p(t)=\left(p_{0}(t), \ldots, p_{n-1}(t)\right)$ with functions $p_{i-1}, i=1, \ldots, n$ being defined by [12], [13]

$$
p_{0}(t)=\bar{\sigma}_{n}\left(r_{1}, . ., r_{n}\right) c^{n}(t),
$$


and for $j=1, \ldots, n-1$,

$$
\begin{aligned}
p_{j}(t)= & \frac{(\sqrt{c(t)})^{n-j}}{\left(c_{0} T\right)^{n-j}} \sum_{k=j}^{n}(-1)^{k-j} \bar{\sigma}_{n-k}\left(r_{1}, \ldots, r_{n}\right)\left(\begin{array}{c}
k-1 \\
j-1
\end{array}\right) \frac{k !}{j !} \\
& \times\left(c_{0} T \sqrt{c(t)}\right)^{n-k}
\end{aligned}
$$

where $c(\cdot)$ is given by (12), $r_{i}>0, r_{i} \neq r_{j}$ for $i \neq j$ in the range of $n$ and $\bar{\sigma}_{n-k}(\cdot)$ are the elementary symmetric polynomials defined by

$$
\begin{aligned}
\bar{\sigma}_{0}\left(r_{1}, \ldots, r_{n}\right) & =1, \\
\bar{\sigma}_{k}\left(r_{1}, \ldots, r_{n}\right) & =\sum_{1 \leq i_{1} \leq i_{2} \leq \ldots i_{k} \leq n} r_{i_{1}} r_{i_{2}} \ldots r_{i_{k}}, \\
\bar{\sigma}_{n}\left(r_{1}, \ldots, r_{n}\right) & =\prod_{i=1}^{n} r_{i},
\end{aligned}
$$

and $\bar{\sigma}_{k}\left(r_{1}, \ldots, r_{n}\right)=0$, for $k \geq n$.

Similar computations to the scalar case prove that the PDE equation of transformation (39) is as follows:

$$
\gamma_{x}(t, x)-\gamma_{t}(t, x)=C(t)^{\top} \gamma(t, x)
$$

where $\gamma$ is defined on $\mathcal{T}_{\gamma}$.

Proposition 4: The system (46) has a well-posed $\mathcal{C}^{\infty}$ solution on $\mathcal{T}_{\gamma}$, given by

$$
\begin{aligned}
\gamma(t, x)= & V^{-\top}(t) \mathbf{D}^{-1}(t) \mathbf{D}(t-h+x) V^{\top}(t-h+x) \\
& \times \gamma(t-h+x, h),
\end{aligned}
$$

where $\mathbf{D}(s)=\operatorname{diag}\left(e^{-r_{1} c_{0} T \sqrt{c(s)}}, \ldots, e^{-r_{n} c_{0} T \sqrt{c(s)}}\right)$

$\gamma_{i}(t-h+x, h)=a_{i-1}-p_{i-1}(t-h+x), \quad i=1, \ldots, n$,

$p_{i-1}$ defined by (41), (42), and $V$ is polynomial-based Vandermonde matrix given as follows:

$$
V(t)=\left(\begin{array}{ccc}
1 & \cdots & 1 \\
\left(\delta^{0}\left(-r_{1} c\right)\right)(t) & \cdots & \left(\delta^{0}\left(-r_{n} c\right)\right)(t) \\
\vdots & \ddots & \vdots \\
\left(\delta^{n-2}\left(-r_{1} c\right)\right)(t) & \cdots & \left(\delta^{n-2}\left(-r_{n} c\right)\right)(t)
\end{array}\right)
$$

with $\left(\delta^{k}\left(-r_{i} c\right)\right)(t)=\frac{-r_{i} c(t)\left(\sqrt{c(t)}^{k}\right.}{\left(c_{0} T\right)^{k}} k ! L_{k}^{(1)}\left(r_{i} c_{0} T \sqrt{c(t)}\right)$, $k=0, \ldots, n-2$, where $L_{k}^{(1)}($.$) are the generalized Laguerre$ polynomials.

\section{A. Prescribed-time predictor control}

From 39, at $x=h$, and using (48), the boundary control is then as follows:

$$
u(t)=\gamma^{\top}(t, h) P\left(X(t)+\int_{0}^{h} \int_{0}^{\sigma} e^{A(y-\sigma)} B(\sigma) d y \omega(t, \sigma) d \sigma\right) .
$$

1) Stability result:

Theorem 2: Let $c$ be given by (12) and let $r_{\min }=$ $\min _{i=1, \ldots, n}\left\{r_{i}\right\}$ with $r_{i}>0$ involved in (41)-(42). Let $h>0, c_{0}>0, T>0$ fixed. Let $\gamma$ be given by (47). Then, the solution of the closed-loop system (37) with prescribed-time predictor control (50) is UPrTS in the following sense: for any $X_{0} \in \mathbb{R}^{n}$ and $\omega(0, \cdot) \in L^{2}((0, h), \mathbb{R})$, the quantity $\|X(t)\|^{2}+\|\omega(t, \cdot)\|_{L^{2}}^{2}$ remains bounded for $t \in[0, \max \{T, h\}]$, and for all $t \in[\max \{T, h\}, T+h)$, the following estimate holds:

$$
\begin{aligned}
\|X(t)\|^{2}+\|\omega(t, \cdot)\|_{L^{2}}^{2} \leq & M e^{-2 r_{\min } c_{0} T \sqrt{c(t)}}\|\gamma(t, \cdot)\|_{L^{2}}^{2} \\
& \times\left(\left\|X_{0}\right\|^{2}+\|\omega(0, \cdot)\|_{L^{2}}^{2}\right),
\end{aligned}
$$

with for some constant $M>0$. In particular,

$$
\|X(t)\|^{2}+\|\omega(t, \cdot)\|_{L^{2}}^{2} \rightarrow 0, \quad \text { as } \quad t \rightarrow T+h .
$$

Moreover, $|u(t)| \rightarrow 0$ as $t \rightarrow T$.

\section{Simulations}

We consider a scalar linear equation with distributed input delay (2), with $a=2, b=0.5, c_{0}=2$ and $h=1 \mathrm{~s}$. We fix $T=4 \mathrm{~s}$. Numerical simulations were done by discretizing the cascade PDE-ODE system (3) and making use of transformation (7). Figure 1 shows the evolution of the $L^{2}$-norm of the closed-loop system (plotted in logarithmic scale) with the prescribed-time control $u(t)$ in blue line (see (22)). One can observe convergence to the origin as $t \rightarrow 5 \mathrm{~s}$. Figure 1 shows also the evolution of the $L^{2}$-norm of the closed-loop system in red dashed line using the following predictor feedback for exponential stabilization (see [34], [1]),

$$
u(t)=k\left(X(t)+b \int_{0}^{h} \int_{0}^{\sigma} e^{a(y-\sigma)} d y w(t, \sigma) d \sigma\right),
$$

where, we chose $k=-18.5$ such that $a+b \times k<0$. On the other hand, Figure 2 shows us the evolution of the solution $X(t)$ of the closed-loop system (3), in blue using the prescribed-time control (see (22)), and in red dashed line using the predictor feedback (51) for exponential stabilization. It shows also the evolution of $\omega$ the solution of the transport PDE.

\section{CONCLUSION}

In this paper, we were able to treat the problem of prescribed-time stability of linear systems with distributed input delay. The main ideas are developed for delay compensation of a scalar linear equation with distributed input delay. The prescribed-time predictor feedback design is achieved based on the backstepping approach using a backsteppingforwarding transformation and a reduction-based change of variables. A sketch of the results for a more general case ( $n$-dimesnional LTI systems) is illustrated.

Future work includes prescribed-time observers of LTI systems subject to distributed sensor delays. 


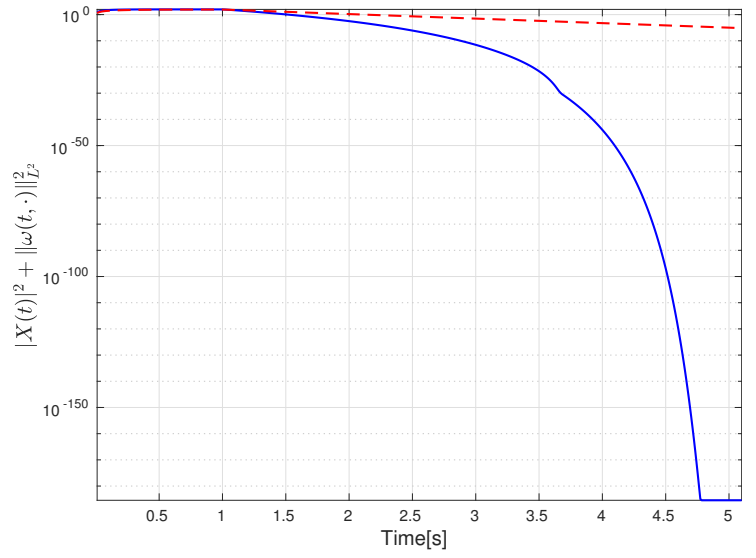

Fig. 1. The evolution of the $L^{2}$-norm of the closed-loop system (3) (logarithmic scale)
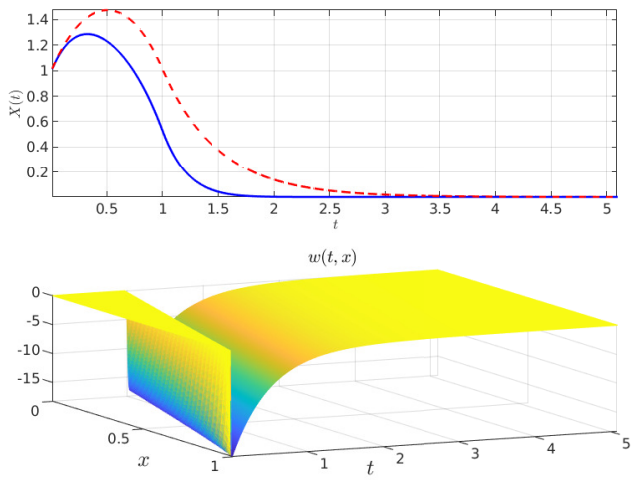

Fig. 2. The evolution of the solutions of the closed-loop system (3) (logarithmic scale), and also the numerical solution of the transport PDE $\omega(t, x)$.

\section{REFERENCES}

[1] M. Bekiaris-Liberis, N.and Krstic. Nonlinear Control Under Nonconstant Delays. Society for Industrial and Applied Mathematics, Philadelphia, PA, 2013.

[2] N. Bekiaris-Liberis and M. Krstic. Lyapunov stability of linear predictor feedback for distributed input delays. IEEE Transactions on Automatic Control, 56(3):655-660, 2011.

[3] N. Bekiaris-Liberis and M. Krstic. Lyapunov stability of linear predictor feedback for distributed input delays. IEEE Transactions on Automatic Control, 56(3):655-660, 2011.

[4] S.P. Bhat and D.S. Bernstein. Finite time stability of continuous autonomous systems. SIAM J. Control Optim., 38(3):751-766, 2000.

[5] D. Bresch-Pietri and M. Krstic. Delay-adaptive predictor feedback for systems with unknown long actuator delay. IEEE Transactions on Automatic Control, 55(9):2106-2112, 2010.

[6] D. Bresch-Pietri and M. Krstic. Delay-adaptive control for nonlinear systems. IEEE Transactions on Automatic Control, 59(5):1203-1218, 2014.

[7] J.-M. Coron, L. Hu, and G. Olive. Finite-time boundary stabilization of general linear hyperbolic balance laws via fredholm backstepping transformation. Automatica, 84:95-100, 2017.

[8] J.-M. Coron and H.-M. Nguyen. Null controllability and finite time stabilization for the heat equations with variable coefficients in space in one dimension via backstepping approach. Archive for Rational Mechanics and Analysis, 225(3):993-1023, 2017.

[9] J. Deutscher. Finite-time output regulation for linear $2 \times 2$ hyperbolic systems using backstepping. Automatica, 75:54 - 62, 2017.
[10] D. Efimov, P. Polyakov, E. Fridman, W. Perruquetti, and J.-P. Richard Comments on finite-time stability of time-delay systems. Automatica, 50(7):1944 - 1947, 2014.

[11] R. Engel and G. Kreisselmeier. A continuous-time observer which converges in finite time. IEEE Transactions on Automatic Control, 47(7):1202-1204, 2002.

[12] N. Espitia and W. Perruquetti. Prescribed-time predictor control of LTI systems with input delay. In Accepted for presentation to the 59th IEEE Conference on Decision and Control (CDC), Jeju Island, Republic of Korea, 2020.

[13] N. Espitia and W. Perruquetti. Predictor-feedback prescribed-time stabilization of LTI systems with input delay. IEEE Transactions on Automatic Control, 2021.

[14] N. Espitia, A. Polyakov, D. Efimov, and W. Perruquetti. Boundary time-varying feedbacks for fixed-time stabilization of constantparameter reaction-diffusion systems. Automatica, 103:398 - 407, 2019.

[15] V.T. Haimo. Finite time controllers. SIAM Journal of Control and Optimization, 24(4):760-770, 1986.

[16] J. Holloway and M. Krstic. Prescribed-time observers for linear systems in observer canonical form. IEEE Transactions on Automatic Control, 64(9):3905-3912, 2019.

[17] J. Holloway and M. Krstic. Prescribed-time output feedback for linear systems in controllable canonical form. Automatica, 107:77-85, 2019.

[18] I. Karafyllis. Finite-time global stabilization by means of time-varying distributed delay feedback. SIAM J. Control Optim., 45:320-342, 2006.

[19] P. Krishnamurthy, F. Khorrami, and M. Krstic. A dynamic high-gain design for prescribed-time regulation of nonlinear systems. Automatica, 115:108860, 2020.

[20] M. Krstic. Delay Compensation for Nonlinear, Adaptive, and PDE Systems. Birkhäuser, 2009.

[21] M. Krstic and A. Smyshlyaev. Backstepping boundary control for first-order hyperbolic PDEs and application to systems with actuator and sensor delays. Systems \& Control Letters, 57(9):750-758, 2008.

[22] F. Lopez-Ramirez, A. Polyakov, D. Efimov, and W. Perruquetti. Finite-time and fixed-time observer design: Implicit lyapunov function approach. Automatica, 87:52 - 60, 2018.

[23] F. Mazenc, M. Malisoff, and S.-I. Niculescu. Reduction model approach for linear time-varying systems with delays. IEEE Transactions on Automatic Control, 59(2068-2081), 2014.

[24] W. Michiels and B. Zhou. On the fixed-time stabilization of input delay systems using act-and-wait control. Systems \& Control Letters, 146, 2020.

[25] E. Moulay, M. Dambrine, N. Yeganefar, and W. Perruquetti. Finitetime stability and stabilization of time-delay systems. Systems \& Control Letters, 57:561-566, 2008.

[26] A. Polyakov, D. Efimov, and W. Perruquetti. Finite-time and Fixedtime Stabilization: Implicit Lyapunov Function Approach. Automatica, 51(1):332-340, 2015

[27] Y.-D. Song, Y.-J. Wang, J.-C. Holloway, and M. Krstic. Timevarying feedback for regulation of normal-form nonlinear systems in prescribed finite time. Automatica, 83:243 -251, 2017.

[28] D. Steeves, M. Krstic, and R. Vazquez. Prescribed-time H1stabilization of reaction-diffusion equations by means of output feedback. In 2019 18th European Control Conference (ECC), pages 1932 1937, 2019.

[29] D. Tran and T. Yucelen. Finite-time control of perturbed dynamical systems based on a generalized time transformation approach. Systems \& Control Letters, 136:104605, 2020.

[30] X. Xu, L. Liu, and G. Feng. Consensus of discrete-time linear multiagent systems with communication, input and output delays. IEEE Transactions on Automatic Control, 63(2):492-497, 2017.

[31] B. Zhou. Finite-time stabilization of linear systems by bounded linear time-varying feedback. Automatica, 113:108760, 2020.

[32] B. Zhou, H. Gao, Z. Lin, and G.-R. Duan. Stabilization of linear systems with distributed input delay and input saturation. Automatica, 48(5):712-724, 2012

[33] B. Zhou, Z.-Y. Li, and Z. Lin. Observer-based output feedback control of linear systems with input and output delays. Automatica, 49(7):2039 $-2052,2013$.

[34] Y. Zhu, M. Krstic, and H. Su. Delay-adaptive control for linear systems with distributed input delays. Automatica, 116:108902, 2020. 